\title{
Identification of possible factors influencing temperatures elevation during implant site preparation with piezoelectric technique
}

\author{
Luca Lamazza, DMD, PhD ${ }^{1}$ \\ Domenica Laurito, DDS ${ }^{1}$ \\ Marco Lollobrigida, DDS ${ }^{1}$ \\ Orlando Brugnoletti, MD, DDS ${ }^{1}$ \\ Girolamo Garreffa, MP2 \\ Alberto De Biase, MD, DS 1 \\ ${ }^{1}$ Oral and Maxillo-Facial Sciences Department, \\ "Sapienza" University of Rome, Italy \\ 2 Euro-Mediterranean Institute of Science and Tech- \\ nology (I.E.ME.S.T.), Palermo, Italy; Mediterranean \\ Diagnostic (ME.DI.), Castellammare di Stabia, \\ Napoli, Italy
}

Corresponding author:

Alberto De Biase, MD, DS

Oral and Maxillo-Facial Sciences Department, "Sapienza" University of Rome, Italy

E-mail:alberto.debiase@uniroma1.it

\section{Summary}

Background. Overheating during implant site preparation negatively affects the osseointegration process as well the final outcome of implant rehabilitations. Piezoelectric techniques seem to provide to a gentle implant preparation although few scientific reports have investigated the heat generation and its underlying factors.

Purpose. To investigate, through a proper methodological approach, the main factors influencing temperature rise during piezoelectric implant site preparation.

Materials and methods. Different piezoelectric tips (IM1s, IM2, P2-3, IM3, Mectron Medical Technology, Carasco, Italy) have been tested. The experimental set-up consisted in a mechanical positioning device equipped with a load cell and a fluoroptic thermometer.

Results. The first tip of the sequence (IM1s) generated the highest temperature increasing $(\Delta T)$. The diamond tips (IM1s and P2-3) determined higher $\Delta \mathrm{T}$ values than the smooth tips (IM2 and IM3). Further tests with IM1s suggested that the temperature elevation during the first thirty seconds may be predictive of the maximal temperature as well as of the overall thermal impact.

Conclusions. Working load, working movements management and bone features resulted to be the main factors influencing temperature rise during piezoelectric implant site preparation. Irrigant temperature and clogging effect may also synergically contribute to the heat generation.

Key words: implant site preparation, piezosurgery, heat.

\section{Introduction}

Overheating during implant site preparation has been recognized to be one of the main factors for early implant failure (1). Due to the low bone thermal conductivity, heat generated by osteotomies is not effectively dissipated and tends to remain within the surrounding tissue increasing the possibility of thermal injury (2). Keeping in mind this unfavorable situation, several authors stressed the need for "atraumatic" implant site preparations (3-5), taking into account both mechanical and thermal damage of bone. However, such an assertion has to deal with the real possibility to cut bone without causing any alteration that may compromise the healing process. The term "atraumatic", instead of an absence of trauma, should rather refer to a limited damage that reasonably will not impede a successful osseointegration. It is worthy to note, in fact, that implant osseointegration is the overall result of a bone healing process initiated by the surgical trauma.

In this panorama, piezoelectric techniques have been proposed as an alternative method for implant site preparation. In 2000 , Vercellotti (6) described piezoelectric technique as a novel device to perform osteotomies and osteoplasties in oral surgery. Two principal features characterize piezoelectric devices: micrometric cut and selective cut with soft tissue preservation (7). Histological and biochemical studies reported promising results about bone healing after piezoelectric bone surgery (810) although the thermal effects are still considered a critical aspect of the interaction between ultrasound devices and tissues $(11,12)$.

Heat is defined as a process in which energy flows from hot to cold objects. Despite the simple definition, heat transfer is an extremely complex physical phenomenon to analyze. A great deal of research has been expended to measure heat production during bone cutting with drilling techniques (13). Several important issues arise when dealing with temperature recording in bone tissue concerning the measuring device, the distance of the thermometer probe from the heat source, the cooling system and the thermal properties of bone (e.g. type and shape of bone samples, thermal conductivity and heat capacity). To overcome the limits related to the large number of 
factors at stake, a proper methodological approach and dedicated technical environment is essential.

In a previous report (14), an alternative method was proposed to record temperatures during implant site preparation with different drills in bovine bone. The use of a fluoroptic thermometer revealed to be a reliable method for real-time data recording. Notwithstanding, concerns remained about the probe position reproducibility (measurement point) as well as the working load applied by the operator's hand. A dedicated mechanical device has been designed to obtain the reproducibility of the measurement point and to control the most important working parameters. The present study aims to identify main factors and phenomena that are or could be responsible for temperature elevation during implant site preparation with piezoelectric technique.

\section{Materials and methods}

\section{Bone samples}

The study specimens consisted of bovine bone ribs, sectioned in blocks measured approximately $6 \times 4 \times 3 \mathrm{~cm}$. Bovine ribs represent a well established bone model for the human mandible due to similarities in bone density, the ratio between cortical and cancellous bone and thermal conductivity $(2,15-18)$. Density analysis demonstrated similar Hounsfield values between human cortical and cancellous bone and cortical and cancellous bovine bone. Hounsfield units of cortical bone in an average human mandible have been observed to be 1400 to 1600 with a medullary of 400 to 600 Hounsfield units. The cortical bone in bovine ribs has been demonstrated to be 1400 Hounsfield units and the medullary bone 470 Hounsfield units (19). According to Sedlin and Hirsch guidelines (20), the bone samples were kept wet at all times, stored frozen in saline at $-10^{\circ} \mathrm{C}$, and used within 3 to 4 weeks.

\section{Mechanical Positioning Device (MPD)}

MPD is a micrometer-controlled tridimensional positioning device (Fig. 1), originally designed to provide a steady measurement point regardless of the bone sample shape and to reproduce handpiece movements similar to those in the clinical practice.

The device basically consists of three positioning stages incorporated into a highly rigid and crushproof structure. By the action of micrometer screws, it is possible to obtain three-dimensional movements of both the study sample and the drill used to create the holes for the thermometer sensors. The holes had a diameter of $0.6 \mathrm{~mm}$ and were drilled $2 \mathrm{~mm}$ down from the top of the sample to a depth of $2 \mathrm{~mm}$. This ensured temperature detection within the cortical layer. MPD provided a desired distance of $(0.5 \pm 0.05) \mathrm{mm}$ between the thermometer sensors and the tips; this distance was calibrated, for IM1s, on the tip surface at $9 \mathrm{~mm}$ in length and on the tip maximum diameter for the other tips.

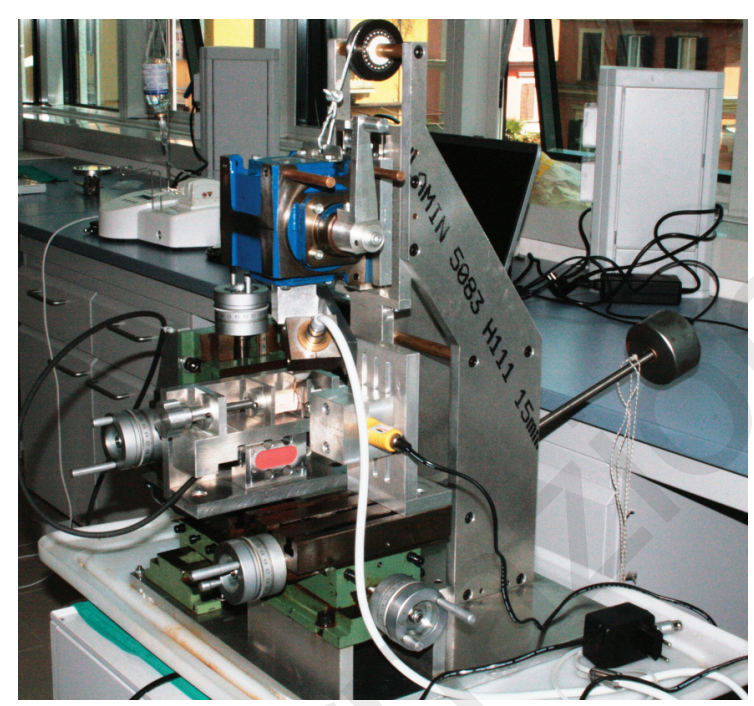

Figure 1. Micrometer-controlled tridimensional positioning device (MPD).

The piezoelectric handpiece was secured to an angular transmission tool free to slide longitudinally. The longitudinal movement was coupled with a rotational movement by moving a handle. This combined motion was manually powered by an operator.

A load cell equipped with a display showed in real time the applied load on bone in order to control and maintain it within pre-determined ranges.

\section{Thermometer}

The acquisition and recording of temperature data were performed by using an optic thermometry system (Luxtron m 3300 Biomedical Lab Kit, Luxtron Corporation, Santa Clara, CA, United States) consisting of a principal unit and four non-metallic probes of $0.5 \mathrm{~mm}$ diameter. The instrument measures temperatures in a range of 0 to $+120^{\circ} \mathrm{C}$ with a response time of $0.25 \mathrm{sec}$ using a patented fluoroptic technology based on a temperature sensitive phosphorescent sensor located at the end of the optical fibers. Pulses of light transmitted down the fiber optic probe cause the sensor excitation. The instrument detects and calculates the decay time of fluorescence after each pulse. The decaying light signal returns through the fiber to the instrument where it is processed by converting the analogue signal into a digital value, which is then converted into a calibrated and corrected temperature. Data are then recorded and graphed using a dedicated software (TrueTemp, LumaSense Technologies, Inc., Santa Clara, CA, United States).

\section{Tests description}

Two different sets of tests have been performed in the study. In the first set (40 osteotomies to a depth of $9 \mathrm{~mm}$ ) IM1s, IM2, P2-3, IM3 tips (Mectron Medical 
Identification of possible factors influencing temperatures elevation during implant site preparation with piezoelectric technique

Technology, Carasco, Italy) were tested maintaining working loads in predetermined displayed ranges (50$150 \mathrm{gr}$ for IM1s and 200-500 gr for the other tips). IM1s and P2-3 are diamond coated tips with diameter of $2 \mathrm{~mm}$ and 2 to $3 \mathrm{~mm}$ respectively. IM2 and IM3 are smooth shank tips with an end cut designed to enlarge the initial osteotomy performed with IM1s. P2-3 tip is dedicated to a coronal preparation after IM2 and before IM3. Differently from IM2, IM3 and P2-3, IM1s is equipped with an external water cooling system. The piezoelectric unit (Piezosurgery, Mectron Medical Technology, Carasco, Italy) was set in bone mode with special level power for IM1s and implant mode for the other tips. A cooling saline solution at room temperature with a flow rate of $28 \mathrm{~mL} / \mathrm{min}$ was used. One operator manually managed the handpiece movements. The osteotomies were obtained through an alternation of working cycles. Each cycle included one longitudinal action within a predetermined working load followed by a rotation action without load. Working cycles were completed by the tip uplift phase interrupting any bone tip contact (Fig. 2).
For each bone sample 3 osteotomies spaced $6 \mathrm{~mm}$ were carried out. One thermometer probe was subsequently moved in prepared holes corresponding to the osteotomies thus obtaining 3 separate measurements. The second set of data was collected with IM1s solely. Differently from the first set, the experimental setup involved the use of 4 fibers in different measurement points. A silicon heat compound sealing the boundary of canal was used to isolate fiber sensors from cooling solution and outer environment. Three measurement points were prepared with a $6 \mathrm{~mm}$ linear inter-distance. The fourth point was prepared down from the middle point to detect the bulk sample temperature then not directly related to the tip action. This set-up usefully provided different measurement conditions: 1) two adjunctive symmetric detections when drilling on the middle point and 2) two adjunctive asymmetric detections when drilling on the left-most or rightmost point (Fig. 3). In the former, the further measurement points are both $6 \mathrm{~mm}$ from the main test point whereas in the latter are at 6 and $12 \mathrm{~mm}$, respectively. The drilling order was randomly followed.

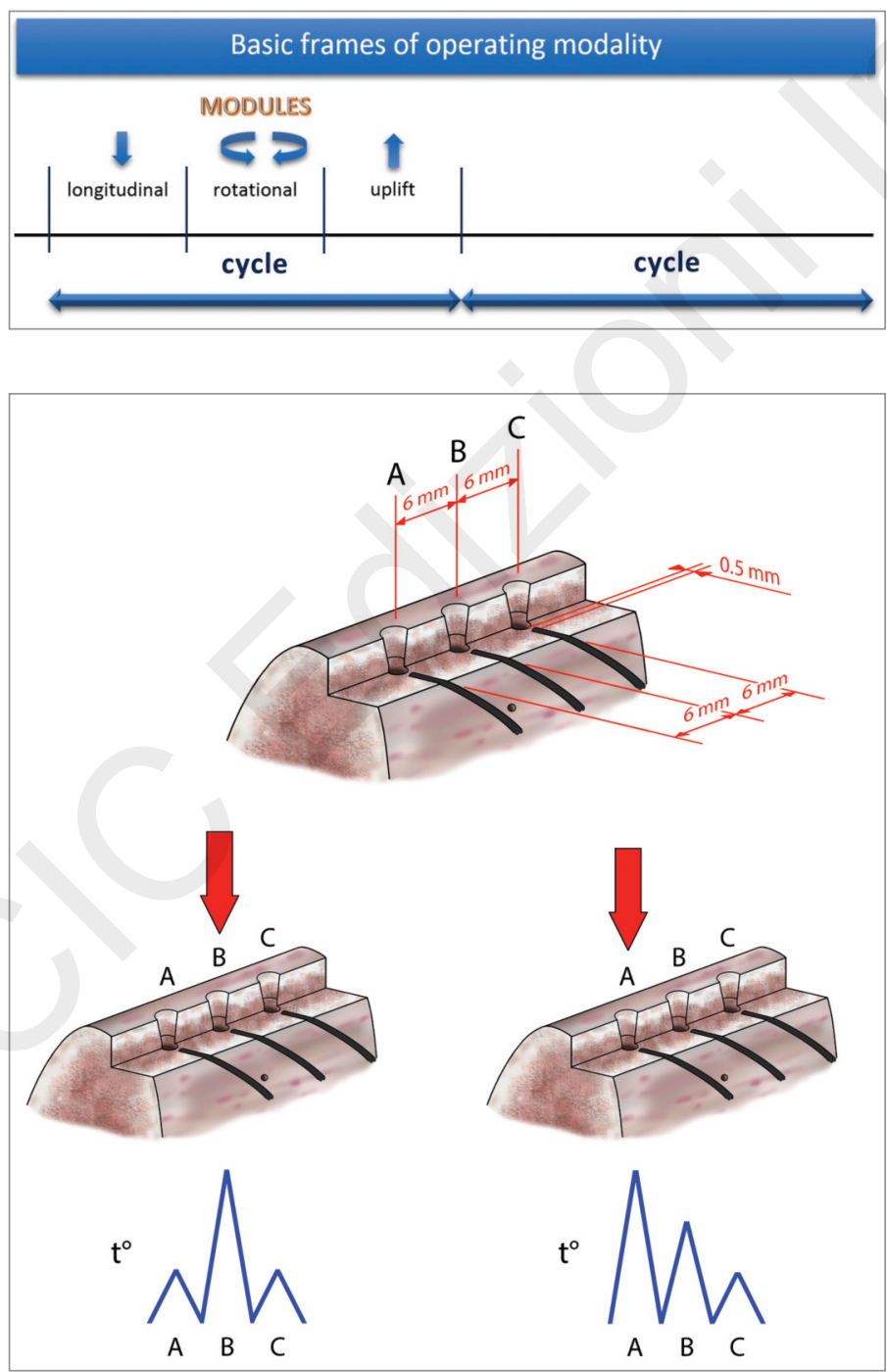

Figure 2. Drawing of the adopted working cycle with operative phases of action.

Figure 3. Methodological approach: symmetrical (down left) and asymmetrical (down right) conditions of temperature detection by random drilling of sites spaced $6 \mathrm{~mm}$. 
All tests have been conducted at a room temperature $\left(18-24^{\circ} \mathrm{C}\right)$. After a slide section of bone samples, radiographs were taken to check the distance for all tests. Further tests were also performed varying some conditions as described in the results and discussion section.

\section{Analytical parameters}

For the first set of data two parameters have been considered:

- $\Delta T_{\max }$ : temperature variation from the baseline to the maximum value;

- $\Delta \mathrm{T}_{30}$ : temperature variation from the baseline to the temperature value recorded at 30 seconds.

For the second set of measurements, further parameters were introduced and defined as following:

- $\quad \sum N_{\text {max }}$ : sum of temperature values sampled each second over the time period $\Delta t$, where $\Delta t$ is the time elapsed from the on-set to the maximum recorded temperature value;

- $\quad \sum N_{\max } / \Delta t_{m}$ : the value of the parameter defined above divided by $\Delta t_{m}$; it represents the mean temperature value on the time period $\Delta t_{m}$;

- $\quad \sum \mathrm{N}_{\mathrm{f}}$ : sum of temperature values sampled each second over the time period $\Delta \mathrm{t}_{\mathrm{f}}$, where $\Delta \mathrm{t}_{\mathrm{f}}$ is the time elapsed from the test on-set to the end;

- $\quad \sum N_{f} / \Delta t_{f}$ : the value of the parameter defined as above but divided by $\Delta \mathrm{t} f$; it represents the mean temperature value over the entire time period $\Delta \mathrm{t}_{\mathrm{f}}$;

- $\quad \sum N_{30}$ : sum of temperature values sampled each second over the first 30 seconds.

All temperature measurements were reported on graphs with their time course values (1 second time scale); the average temperature of the 20 seconds before the test onset was assumed as baseline value. Each of the above parameters if appears with a dash over the symbol represents an average value, for ex-

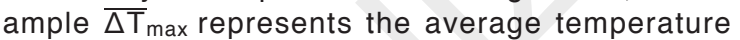
variation from the baseline to the maximum value.

\section{Results and discussions}

The present research sought to identify the factors that could be responsible of temperature rise during the procedure of implant site preparation with piezoelectric technique. The MPD ensured a fixed distance of $(0.5 \pm 0.05) \mathrm{mm}$ between the detecting fiber and the tip surface (measurement point) regardless to the sample shape. The distance reproducibility of the measurement point was confirmed by radiographic inspection. Furthermore, MPD was designed to provide dynamically applied loads and handpiece movements as in the clinical practice (longitudinal and rotational). A fluoroptic thermometer was used to improve the accuracy of temperature measurements and to avoid those difficulties generally encountered using thermocouples, e.g. the need for calibration and possible intrinsic measurement errors (21).

Expecting higher values than in cancellous bone, all temperatures measurements have been performed within the cortical layer (22). However, different literature data have been reported on this issue (23-25). Stelzle et al. (26) observed a highest thermal impact in the cortical bone with both piezoelectric and rotating techniques supporting the hypothesis that cortical bone is more susceptible of temperature rise than cancellous bone.

In the first set of osteotomies the highest mean values for $\Delta T_{\max }$ occurred with IM1s (Tab. 1). Mean temperature values detected for the diamond coated tips (IM1s and P2-3) resulted higher than for the smooth tips (IM2 and IM3). Nevertheless, the restricted cutting portions of IM2 and IM3 may also contribute to reduce contact surface and frictional heating. P2-3, despite the diamond coating, presents lower values than IM1s. This can be ascribed to the shorter active portion of P2-3 (less contact surface) and to its peculiar action consisting in a simple flaring of the osteotomy obtained with IM2. In addition, the lower mean values of $\Delta T_{\max }$ of IM2, P2-3 and IM3 may arise from their internal irrigation system.

These preliminary tests suggest IM1s to be the tip generating the greater amount of heat. Differently from IM2, P2-3 and IM3, IM1s encounters compact bone without predrilling. Moreover, compared to the other tips, IM1s has an external irrigation. The external irrigation plays an important role in the early working phases of IM1s, as indicated by the $\Delta T_{30}$ values (similar to the tips with internal irrigation), but may result inadequate in the deepest portions of preparation, where the greater amount of heat is probably produced. A reduced irrigation efficiency could enhance the clogging effect due to ineffective debris removal. A possible compaction of a bone remains, determined by ultrasounds action, reduces cutting performance, inducing the operator to increase the applied load. The result will consist in an inevitable temperature rise.

While the first tests set aimed to detect the maximum temperatures, the second set, focusing on IM1s, was carried out to identify the main factors influencing temperature elevation. These measurements were performed on two different bone samples (three for each one) as showed in Table 2.

Differently from the preliminary tests, the overall duration of the single trial was dilated of about 2-3 times to obtain an apparent time resolution suitable to visualize the temperature line shapes related to the single working phases (Fig. 3). Regular working movements were also attempted.

The simultaneous temperature measurements (time courses) related to the four test points in one IM1s

Table 1. Mean temperature variations from the baseline to the maximum value $\left(\overline{\Delta \mathrm{T}}_{\max }\right)$ and from the baseline to the temperature at 30 seconds $\left(\overline{\Delta \mathrm{T}}_{30}\right)$ for each tip.

\begin{tabular}{lll}
\hline & $\overline{\mathbf{\Delta T}}_{\max }$ & $\overline{\mathbf{\Delta T}}_{\mathbf{3 0}}$ \\
\hline IM1s & 19,13 & 7,71 \\
IM2 & 7,45 & 5,57 \\
P2-3 & 8,42 & 7,02 \\
IM3 & 8,22 & 7,15 \\
\hline
\end{tabular}


Identification of possible factors influencing temperatures elevation during implant site preparation with piezoelectric technique

test is presented in Figure 4. A chevron-like pattern in this test is clearly visible and seems to be strictly related to the sequence of longitudinal-rotational actions and pauses. Differences in maximum temperature values and the time in which they occur increase with distance from the operating zone that behaves, for a given period, as a "heat source". These findings were almost constantly observed in all the tests.

Some correlations (Fig. 5) between parameters can be noted. In particular, $\Delta T_{30}$ values seem to be correlated with both $\Delta T_{\max }(\rho=0,88)$ and $\sum N_{f} / \Delta t_{f}(\rho=0,92)$. This suggests that the temperature elevation in the first thirty seconds is somehow predictive of the maximal temperature reached during the osteotomies as well as of the overall thermal impact for each second. It is also reasonable that the adopted operating modality may facilitate these correlations.

Many factors can affect temperatures during implant site surgical preparation. A large number of scientific papers have been published on temperature rise with rotating technique. Speed, pressure, irrigation and bur shape have been identified as some of the most important factors influencing temperatures rising (3). The impact of pressure and irrigation has been also investigated with respect to implant site preparation with piezoelectric instruments $(26,27)$.

The main factors influencing temperatures should be related to: 1) operating modalities; 2) bone sample features.

\section{Operating modality}

In relationship with operating modality, working load, irrigation and management of basic frame modules for each cycle seem to play active role.

Table 2. Parameters used for an objective discussion and identification of critical factors with IM1s.

\begin{tabular}{|c|c|c|c|c|c|c|c|c|}
\hline & & $\begin{array}{l}\Delta \mathrm{T}_{\max } \\
\left({ }^{\circ} \mathrm{C}\right)\end{array}$ & $\begin{array}{l}\sum \mathrm{N}_{\max } \\
\left({ }^{\circ} \mathrm{C} \cdot \mathrm{s}\right)\end{array}$ & $\begin{array}{l}\sum N_{\max } / \Delta t_{m} \\
\left({ }^{\circ} \mathrm{C}\right)\end{array}$ & $\begin{array}{l}\sum N_{f} \\
\left({ }^{\circ} \mathrm{C} \cdot s\right)\end{array}$ & $\begin{array}{l}\sum N_{f} / \Delta t_{f} \\
\left({ }^{\circ} \mathrm{C}\right)\end{array}$ & $\begin{array}{l}\Delta \mathrm{T}_{30} \\
\left({ }^{\circ} \mathrm{C}\right)\end{array}$ & $\begin{array}{l}\sum \mathrm{N}_{30} \\
\left({ }^{\circ} \mathrm{C} \cdot \mathrm{s}\right)\end{array}$ \\
\hline Bone sample & Test 1 & 14,26 & 859,52 & 6,87 & 1592,69 & 8,38 & 2,62 & 58,26 \\
\hline \multirow[t]{2}{*}{ A } & Test 2 & 14,95 & 1095,76 & 6,33 & 1623,23 & 7,37 & 3,73 & 88,16 \\
\hline & Test 3 & 16,52 & 1216,44 & 9,15 & 1697,84 & 9,81 & 4,23 & 86,13 \\
\hline Bone sample & Test 4 & 20,19 & 1336,91 & 10,61 & 2323,97 & 11,43 & 5,59 & 105,46 \\
\hline \multirow[t]{2}{*}{ B } & Test 5 & 31,81 & 1212,99 & 10,83 & 2698,31 & 14,2 & 6,43 & 107,96 \\
\hline & Test 6 & 22,19 & 2001,37 & 13,17 & 2514,27 & 12,21 & 5,58 & 101,70 \\
\hline
\end{tabular}
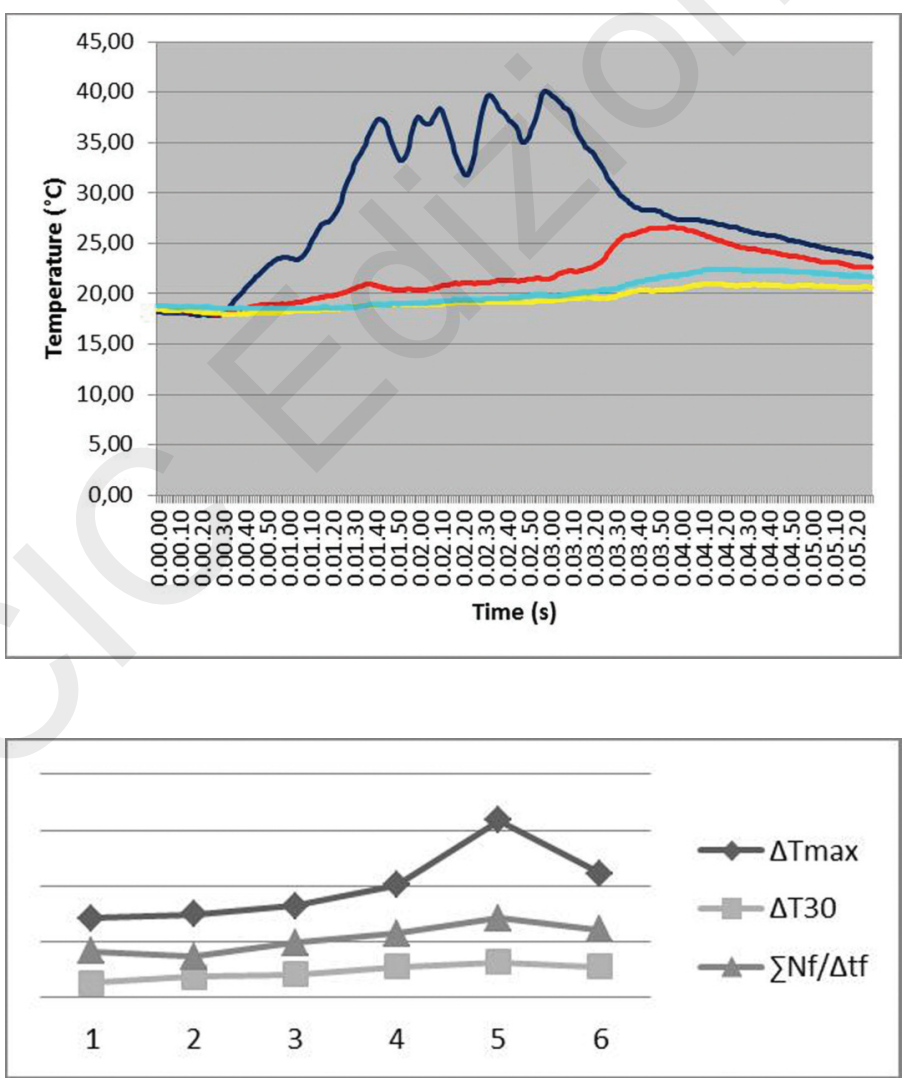

Figure 4. IM1s test with differences in temperature values at different test points. The blue line detect temperature variation in correspondence of site $A$; the red line is referred to the point $B$; cyan line to the point $C$ and yellow line to the point $O$.

Figure 5. Parameters correlations in the test results reported in Table 2 . 


\section{Working load}

The working load is related to the pressure applied by the operator's hand. In the experimental conditions the tip was perpendicular to the bone surface in order to avoid lateral forces allowing an accurate measurement by the load cell, whereas in the clinical context it is clearly difficult to guarantee this condition.

It has been documented that higher working loads induce a higher temperature elevation $(26,28)$. The working load can affect temperature elevation in different ways such as increasing the efficiency of real contact area and thermal coupling between tip and bone surface. Furthermore, a less controlled operating mode $(29,30)$ could determine a variation of the optimal ultrasound operating frequency with a possible thermal energy release in the body tip. Basing on this hypothesis, the piezoelectric tip could become an adjunctive source of heat.

\section{Irrigation}

Although the irrigation was not a specific issue of this investigation, it was proven to play an important role in reducing temperatures $(22,27)$.

However, the irrigation efficacy in limiting temperature elevation is evident in tests with warmed bone samples $\left(37^{\circ} \mathrm{C}\right)$ (Fig. 6). Differential values seem not to change significantly as well as the absolute value reached. This is related to the clear lowering of overall baseline values during irrigation. In these tests red line is the nearest test point, blue and yellow refer to the symmetrical test points. Interestingly, the outermost test point (cyan) follows an almost-Newtonian cooling law and all test points shows an instantaneous spatial thermal equilibrium of the sample in the left and right part of the graph. In these particular tests, performed with IM1s, the irrigant solution was maintained at room temperature with a flow rate of 28 $\mathrm{mL} / \mathrm{min}$.

\section{Management of basic frame modules}

Piezoelectric handpiece movements can largely affect temperatures elevation although this particular aspect is probably the less investigated in literature. An adequate management of working cycles modules can interrupt the applied load, reduce the bone-tip contact and facilitate the debris removal through the irrigant solution minimizing the clogging effect. Moreover, a well suited sequence of working cycles reduces the contact time and allows the bone to cool down (23). However, bone sample cortical thickness couldn't be discarded in heating analysis (as will be discussed later). The importance of the handpiece movements is highlighted by tests with no working cycles simply contacting the tip to bone (Fig. 7). In these tests, a continuous fixed contact between the tip and the bone surface was maintained applying two different a constant load of $300 \mathrm{gr}$ until a depth of 5 $\mathrm{mm}$. The graph shows a sudden temperature rise, in relation to the applied pressure. These data confirm the importance of the working cycle as well as the potential detrimental effects on the healing process when not adopting it. The sudden temperature elevation suggests that even brief hesitations in the clinical handpiece movements can cause a dramatic temperature rise in the bone tissue. It is very interesting to notice however, the high differences in temperature values between the site operation test point and its nearest neighbor (about 60\%) ascribed to the low cortical bone specific heat (31).

\section{Bone sample features}

Bone sample features should be considered as an important factor of temperature rise during implant site preparation. According to different studies, cortical thickness can influence the thermal bone response during drilling $(31,32)$. Observing the Table 2 , temperature parameters in the tests 1,2 and 3 show globally lower average values of measurement

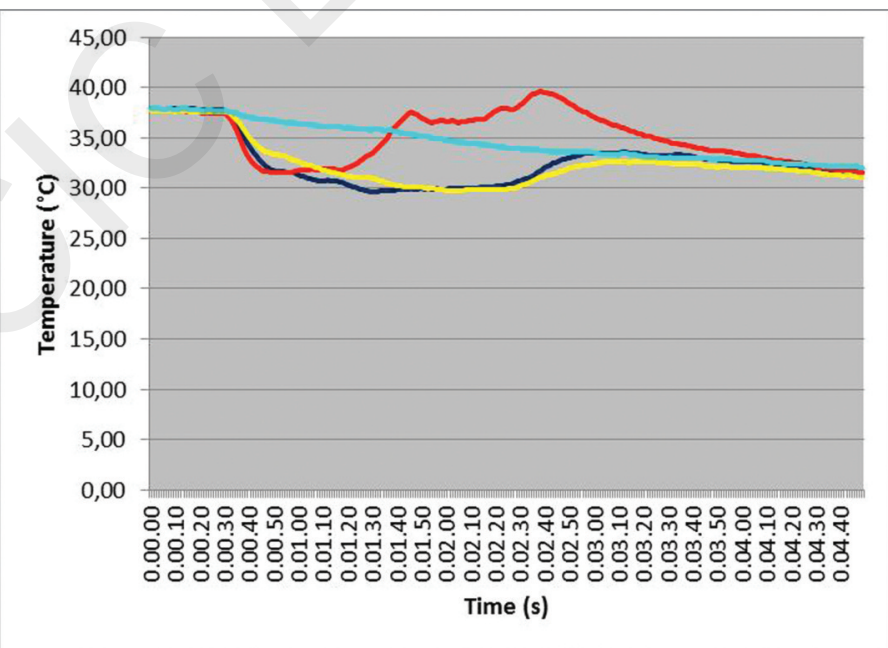

Figure 6. Effect of cooling irrigation in a warmed bone sample. 
Identification of possible factors influencing temperatures elevation during implant site preparation with piezoelectric technique

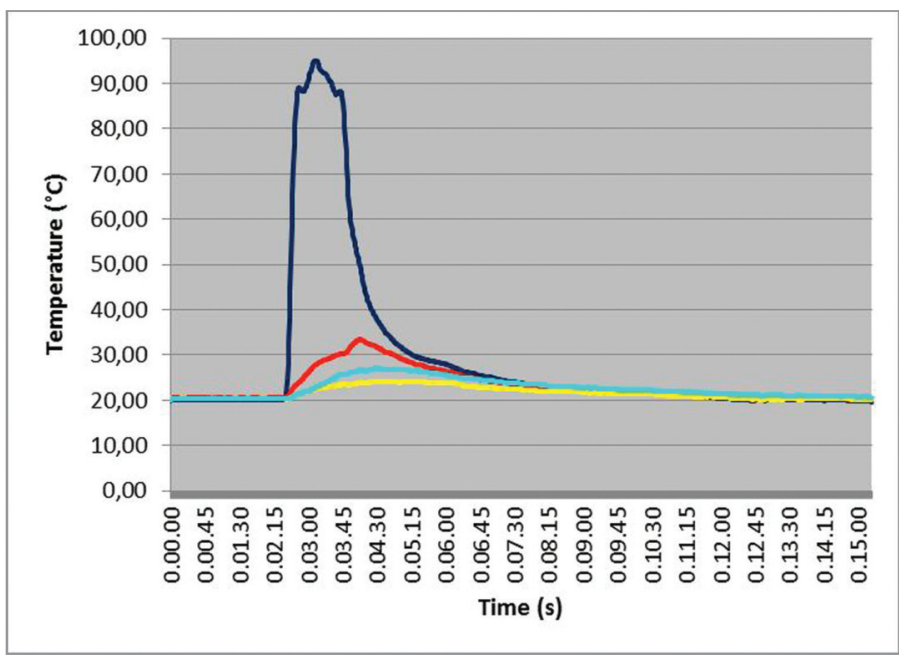

Figure 7. IM1s tests with no working cycle. High temperatures measured at a preparation deep of $5 \mathrm{~mm}$.

parameters compared to tests 4,5 and 6 . This seems to indicate that bone characteristics could not be discarded in the heat production. Assuming that bone characteristics, cortical mineralization and thickness, strongly affect heat production, higher average values, already for $\Delta T_{30}$, should be expected, in bone sample $A$, as occurred in our data. Furthermore, a thicker cortical implies a wider contact tip surface, a longer contact time and an overall energy consumption.

However, it is still an issue of debate whether higher temperatures arise in cortical rather than in cancellous bone (24). The higher compactness of the cortical bone, requiring higher energies and more time for preparation, was pointed out in explanation of different thermal alteration reported in cortical and cancellous bone (26). Probably, different experimental conditions as well as the different thermal properties of cortical and cancellous bone $(33,34)$ can explain the discordance of literature results.

\section{Conclusions}

The present in vitro study has been conducted to investigate the potential factors affecting temperature elevation in piezoelectric assisted implant site preparation. Three factors, among the others, seem to have a broad influence on the heat production: working load, working movements management and bone features. The first tip of the studied sequence (IM1s) presented the most pronounced thermal effects due to the external irrigation and the initial resistance of the cortical layer. Subsequent investigation on IM1s confirmed the importance of the handpiece movements and the applied load to contain temperatures. The indicated threshold level for thermal necrosis of $47^{\circ} \mathrm{C}$ for 1 minute has never been exceeded in the experimental conditions; however, it is to note that a possible necrotic layer produced with the first tip will be removed by the action of the following larger ones. Despite the effort for considering all the possible ele- ments associated with the temperature increase, further investigations must be performed on cortical thickness, irrigant flow rate and temperature, and blood flow effect.

\section{Acknowledgements}

The Authors wish to acknowledge the help provided by Eng. M. Orazi for the project of the MPD; Dr. D. Di Nezio for the graphic illustrations; Mr. A. Pecchia, Mr. A. Chirico, Eng. N. Cerisola and Dr. R. Bocchini for their technical support to the research.

\section{References}

1. Esposito M, Hirsch JM, Lekholm U, Thomsen P. Biological factors contributing to failures of osseointegrated oral implants. (II). Etiopathogenesis. Eur J Oral Sci. 1998;106:721-64.

2. Abouzgia MB, James DF. Temperature rise during drilling through bone. Int J Oral Maxillofac Implants. 1997;12:34253.

3. Eriksson AR, Albrektsson T. Temperature threshold levels for heat-induced bone tissue injury: a vital-microscopic study in the rabbit. J Prosthet Dent. 1983;50:101-7.

4. Tehemar SH. Factors affecting heat generation during implant site preparation: a review of biologic observations and future considerations. Int J Oral Maxillofac Implants. 1999;14:127-36

5. Sutter F, Krekeler G, Schwammberger AE, Sutter FJ. Atraumatic surgical technique and implant bed preparation. Quintessence Int. 1992;23:811-6.

6. Vercellotti T. Piezoelectric surgery in implantology: a case report-a new piezoelectric ridge expansion technique. Int $J$ Periodontics Restorative Dent. 2000;20:358-65.

7. Vercellotti T. Technological characteristics and clinical indications of piezoelectric bone surgery. Minerva Stomatol. 2004:53:207-14

8. Maurer P, Kriwalsky MS, Block Veras R, Vogel J, Syrowatka F, Heiss C. Micromorphometrical analysis of conventional osteotomy techniques and ultrasonic osteotomy at the rabbit skull. Clin Oral Implants Res. 2008;19:570-5.

9. Vercellotti T, Nevins ML, Kim DM, et al. Osseous response 
following resective therapy with piezosurgery. Int $\mathrm{J}$ Periodontics Restorative Dent. 2005;25:543-9.

10. Preti G, Martinasso G, Peirone B, et al. Cytokines and growth factors involved in the osseointegration of oral titanium implants positioned using piezoelectric bone surgery versus a drill technique: a pilot study in minipigs. J Periodontol. 2007;78: 716-22.

11. O'Brien WD Jr. Ultrasound-biophysics mechanisms. Prog Biophys Mol Biol. 2007;93:212-55.

12. Humphrey VF. Ultrasound and matter-physical interactions. Prog Biophys Mol Biol. 2007;93:195-211.

13. Chacon GE, Bower DL, Larsen PE, McGlumphy EA, Beck FM. Heat production by 3 implant drill systems after repeated drilling and sterilization. J Oral Maxillofac Surg. 2006;64:265-9.

14. Laurito D, Lamazza L, Garreffa G, De Biase A. An alternative method to record rising temperatures during dental implant site preparation: a preliminary study using bovine bone. Ann Ist Super Sanita. 2010;46:405-10.

15. Brisman DL. The effect of speed, pressure, and time on bone temperature during the drilling of implant sites. Int $\mathrm{J}$ Oral Maxillofac Implants. 1996;11:35-7.

16. Cordioli G, Majzoub Z. Heat generation during implant site preparation: an in vitro study. Int J Oral Maxillofac Implants. 1997;12:186-93.

17. Davidson SR, James DF. Measurement of thermal conductivity of bovine cortical bone. Med Eng Phys. 2000; 22:7417.

18. Ercoli C, Funkenbusch PD, Lee HJ, Moss ME, Graser GN. The influence of drill wear on cutting efficiency and heat production during osteotomy preparation for dental implants: a study of drill durability. Int J Oral Maxillofac Implants. 2004;19:335-49.

19. Yacker MJ, Klein M. The effect of irrigation on osteotomy depth and bur diameter. Int $\mathrm{J}$ Oral Maxillofac Implants. 1996;11:634-8.

20. Sedlin ED, Hirsch C. Factors affecting the determination of the physical properties of femoral cortical bone. Acta Orthop Scand. 1966;37:29-48.

21. Nell DM, Myers MR. Thermal effects generated by high-intensity focused ultrasound beams at normal incidence to a bone surface. J Acoust Soc Am. 2010;127:549-59.

22. Schutz S, Egger J, Kuhl S, Filippi A, Lambrecht J Th. Intraosseous temperature changes during the use of piezo- surgical inserts in vitro. Int J Oral Maxillofac Surg. 2012;41: 1338-1343.

23. Wachter R, Stoll P. Increase of temperature during osteotomy: in vitro and in vivo investigations. Intl J Oral Maxillofac Surg. 1991;20:245-9.

24. Rashad A, Kaiser A, Prochnow N, Schmitz I, Hoffmann E, Maurer $\mathrm{P}$. Heat production during different ultrasonic and conventional osteotomy preparations for dental implants. Clin Oral Implants Res. 2011;22:1361-5.

25. Sener BC, Dergin G, Gursoy B, Kelesoglu E, Slih I. Effects of irrigation temperature on heat control in vitro at different drilling depths. Clin Oral Implants Res. 2009;20:294-8.

26. Stelzle F, Frenkel C, Riemann M, Knipfer C, Stockmann P, Nkenke E. The effect of load on heat production, thermal effects and expenditure of time during implant site preparation - an experimental ex vivo comparison between piezosurgery and conventional drilling. Clin Oral Implants Res. 2014;25:140-8.

27. Rashad A, Kaiser A, Prochnow N, Schmitz I, Hoffmann E, Maurer $\mathrm{P}$. Heat production during different ultrasonic and conventional osteotomy preparations for dental implants. Clin Oral Implants Res. 2011;22:1361-5.

28. Stelzle F, Neukam FW, Nkenke E. Load-dependent heat development, thermal effects, duration, and soft tissue preservation in piezosurgical implant site preparation: an experimental ex vivo study. Int $\mathrm{J}$ Oral Maxillofac Implants. 2012;27:513-22

29. Cardoni A, MacBeath A, Lucas M. Methods for reducing cutting temperature in ultrasonic cutting of bone. Ultrasonics. 2006;44:37-42.

30. Cardoni A, Lucas M, Cartmell M, Lim F. A novel multiple blade ultrasonic cutting device. Ultrasonics. 2004;42:69-74.

31. Karmani $S$. The thermal properties of bone and the effects of surgical intervention. Current Orthopaedics. 2006;20:52-58.

32. Eriksson RA, Albrektsson T, Albrektsson B. Heat caused by drilling cortical bone: temperature measured in vivo in patients and animals. Acta Orthop Scand. 1984;55:629-31.

33. Clattenburg R, Cohen J, Conner S, Cook N. Thermal properties of cancellous bone. J Biomed Mater Res. 1975; 9:16982.

34. Sean RHD, David FJ. Measurement of thermal conductivity of bovine cortical bone. Medical Engineering \& Physics. 2000;22:741-747. 